\title{
Does time to endoscopic sinus surgery impact outcomes in Chronic Rhinosinusitis? Prospective findings from the National Comparative Audit of Surgery for Nasal Polyposis and Chronic Rhinosinusitis*
}

\author{
C. Hopkins', J. Rimmer², V.J. Lund² \\ ' ENT Dept, Guy's and St Thomas' Hospitals, London, United Kingdom \\ 2 Royal National Throat, Nose and Ear Hospital, London, United Kingdom
}

\author{
Rhinology 53: 10-17, 2015 \\ DOI:10.4193/Rhino13.217 \\ *Received for publication: \\ December 12, 2013
}

Accepted: March 17, 2014

\begin{abstract}
Objectives: Patients with chronic rhinosinusitis refractory to medical management undergo elective surgery. The time from initial diagnosis to surgery varies considerably. The impact of this delay on surgical success has never previously been evaluated.

Design: First-time patients within the National Comparative Audit of Surgery for Nasal Polyposis and Chronic Rhinosinusitis were grouped based on time to surgery: 1) Early cohort: $<12$ months; 2) Mid cohort: 12-60 months; and 3) Late cohort: > 60 months. Co-morbidities and preoperative CT scores were analysed for all patients.
\end{abstract}

Main outcome measures: The 22-item Sino-Nasal Outcome Test scores (SNOT-22) were collected at 0, 3, 12 and 60-months. Absolute and relative SNOT-22 changes from baseline were evaluated.

Results: Asthma and allergies were significantly more prevalent in the Late versus the Early and Mid-cohorts. In addition, patients in the Late cohort had greater symptom burden on the SNOT-22 and more extensive preoperative radiographic disease as determined by Lund-Mackay (LM) scores. SNOT-22 scores demonstrated greater percentage improvements in the Early versus the Midand Late cohorts, at all time points after surgery. At 12 and 60 months after surgery, significantly more patients in the Early group achieved a clinically important change in SNOT-22 scores compared with the other groups. These differences were maintained when cohorts were matched for preoperative co-morbidities.

Conclusion: Patients with asthma and/or allergies are more likely to experience delayed surgical intervention versus other patients. Overall, patients with delayed surgery reported less improvement in SNOT-22 scores than patients treated at earlier time points, regardless of co-morbid status. Delaying surgical intervention may worsen long term clinical outcomes.

Key words: sinusitis, outcomes, sinus surgery

\section{Introduction}

Chronic rhinosinusitis (CRS) is a common condition with a prevalence estimated at $10.9 \%$ (range $6.9-27.1 \%)^{(1)}$. It has significant impact on quality of life ${ }^{(2)}$ and socio-economic burden, with costs per year in the US estimated to be between $\$ 4.3$ and $\$ 5.8$ billion ${ }^{(3)}$. Widely held consensus mandates a trial of maximum medical therapy as the first line of treatment, with surgical intervention reserved only for cases refractory to medical management. The European Position Paper on Rhinosinusitis and Nasal Polyps (EPOS) suggests a number of therapies, including steroid (topical or oral), antibiotics and/or saline irrigation, for at least 12 weeks ${ }^{(4)}$. Failure of medical therapy may thereafter lead 
to surgery. However, there is little in the literature to inform on the optimum timing of surgical intervention beyond that failed three-month time window.

Smith et al. ${ }^{(5,6)}$ in a prospective, non-randomized multi-centered cohort study, offered CRS patients refractory to medical therapy either continued medical management or endoscopic sinus surgery according to patient and surgeon preference. While this inherently introduces bias to the study, this design provides an appropriate reflection on the decision-making process that patients and surgeons may face at time of failed medical therapy. $34 \%$ of the medical management arm did not improve and further worsened within three months of entering the study, and then crossed over into the surgical treatment arm. While these patients experienced significant improvements in patient rated symptom scores, at 12 months' follow-up they had failed to achieve the same level of symptomatic improvement as the cohort who had undergone surgery ab initio. Perhaps delaying surgical intervention may adversely affect outcome in refractory patients. We hypothesise that untreated chronic sinusitis is a progressive disease, and with time patients may develop irreversible changes within the sinus mucosa ${ }^{(7)}$. Early successful treatment may prevent the development of such adverse prognostic characteristics.

In this study, we evaluated whether the duration of symptoms of chronic rhinosinusitis prior to timing of surgical intervention has any impact on the effectiveness of surgery, in terms of symptomatic outcomes. Our hypothesis was that patients with CRS ongoing over a long period of time would be less responsive to surgery than patients treated early on in the natural history of the disease, in terms of a reduction in self-reported outcomes scores.

We could find no evidence in the published literature regarding either the symptomatic or cost effectiveness of surgical interventions at different times after the development of symptoms, and thus performed this study to address these questions. We evaluated patient-reported symptomatic improvements that had been collected prospectively from the National Comparative Audit of Surgery for Nasal Polyposis and Chronic Rhinosinusitis, grouping patients undergoing primary surgery at different time points during the course of their disease. The study methodology and overall results from the Comparative Audit have been previously described ${ }^{(8)}$. However, analysis of results based on patient time to surgery is shown here for the first time.

\section{Materials and methods}

\section{Study design}

The National Comparative Audit of Surgery for Nasal Polyposis and Chronic Rhinosinusitis - a prospective, observational cohort study of 3,128 patients undergoing sinus surgery for CRS in 87 NHS Trusts in England and Wales - has been previously described ${ }^{(8)}$ and included data of patients who underwent mostly maxillary and anterior ethmoidal surgery. The main outcome measure was the Sino-Nasal Outcome Test 22 (SNOT-22), a validated patient reported outcome measure ${ }^{(9)}$. Patients completed the SNOT-22 surveys preoperatively on the day of surgery (baseline) and at 3, 12 and 60 months after surgery.

\section{Setting}

All 156 NHS Trusts in England and Wales were invited to participate to the Audit. A total of 87 centres contributed patients to the study.

\section{Patient inclusion and cohorts}

Patient eligibility criteria and overall selection was described previously ${ }^{(8)}$. For this analysis, only patients undergoing primary surgery were considered, and therefore 1606 patients (51\%) with a prior history of surgery were excluded. Remaining patients who had completed a pre-operative SNOT-22 score were included in this analysis (29 patients were excluded as the baseline SNOT-22 score was missing). Patients were asked to report "How long ago did your nose/sinus symptoms begin". Three cohorts of patients were defined, based on the length of time from onset of sinus symptoms to surgery, as reported by the patient: Early cohort - less than 12 months; Mid cohort - 12-60 months; and Late cohort - more than 60 months of symptoms.

\section{Analyses}

Patient demographics were analysed for each group to identify any potential confounding factors. Disease severity was estimated based on average preoperative Lund Mackay (LM) scores, for all cases where computed tomography (CT) scans were available.

\section{Variables}

Average SNOT-22 scores at each time point were obtained from all patients as previously described, and were calculated and compared to baseline. As rates of asthma were found to be higher in the Late cohort, calculations were repeated after excluding patients with asthma across all groups, to test for a potential confounding effect of this co-morbidity. Similarly, allergies were overly preresented in mid and late cohorts, and calculations were repeated having further excluded patients reporting allergies. The minimally clinically important difference (MCID) in SNOT-22 has been shown to be 8.9. This was used to dichotomise the cohort into two groups; those with improvement in SNOT-22 greater than 8.9, and those failing to achieve this. The percentage of patients achieving the MCID was calculated for each group. Finally, a multiple linear regression was performed to simultaneously control for other demographic 
and operative variables (pre-operative SNOT-22, LM score, age, gender, asthma, allergy and extent of surgery) on the SNOT-22 end point at 60 months.

\section{Statistical analyses}

All statistical analyses were performed on STATA (StataCorp. 2003. Stata Statistical Software: Release 8. College Station, TX: StataCorp LP). Paired t-tests were used to analyse difference in pre- and post-operative scores within groups (significance $a=$ 0.05). One-way analysis of variance (ANOVA) (unequally sized groups) was used to calculate $p$ values of the differences in absolute pre- and post-operative SNOT-22 scores, at all post-operative time points and between all 3 groups. Chi-squared tests of association were used to analyse dichotomized variables.

\section{Results}

A total of 1,493 patients were included in the evaluation, 172 in the Early cohort, 750 in the Mid cohort and 571 in the Late cohort. Response rates declined at each follow-up point. Complete SNOT-22 surveys were available for $80 \%$ of patients at 3 months, $78 \%$ at 12 months, and $49 \%$ at 60 months (Table 1). Patient demographics and clinical presentation at baseline are shown in Table 2. There was a possible association between duration of symptoms and disease severity with patients in the Late cohort showing greater LM scores versus patients in the Early cohort (Late cohort 11.1; Early cohort 9.6; $p=0.05$ ). Similarly the SNOT22 scores of patients in the late cohort were also significantly greater than that of patients in the Early cohort (Late cohort 40.8; Early cohort 35.8; $p<0.006$ ). There was also a significantly greater percentage of patients with asthma and allergies in the Late cohort compared to the Early cohort (Asthma: Late cohort $36.6 \%$, Early cohort 20.1\%, p = 0.02; Allergies: Late cohort 39.2\%, Early cohort $23.1 \%, \mathrm{p}<0.001)$.

Table 1. Response rates for cohort and by duration of symptoms.

\begin{tabular}{|c|c|c|c|c|}
\hline Cohort & $\begin{array}{c}\text { All } \\
\text { patients }\end{array}$ & $\begin{array}{c}\text { Early } \\
\text { Cohort: } \\
<12 \\
\text { months }\end{array}$ & $\begin{array}{c}\text { Mid } \\
\text { Cohort: } \\
12-60 \\
\text { months }\end{array}$ & $\begin{array}{c}\text { Late } \\
\text { Cohort: } \\
>60 \\
\text { months }\end{array}$ \\
\hline $\begin{array}{c}\text { Baseline } \\
\mathbf{n}\end{array}$ & 1493 & 172 & 750 & 571 \\
\hline $\begin{array}{c}3 \text { months } \\
n \\
\text { (\% baseline) }\end{array}$ & $1200(80.4)$ & 144 (83.7) & $602(80.3)$ & 454 (79.6) \\
\hline $\begin{array}{l}12 \text { months } \\
n \\
\text { (\% baseline) }\end{array}$ & 1177 (78.9) & $130(75.6)$ & $594(79.2)$ & $453(79.3)$ \\
\hline $\begin{array}{c}60 \text { months } \\
n \\
\text { (\% baseline) }\end{array}$ & 734 (49.2) & $81(47.1)$ & $375(50)$ & $278(48.7)$ \\
\hline
\end{tabular}

Table 2. Patient demographics by duration of symptoms (CRSwNP - CRS with nasal polyps).

\begin{tabular}{|c|c|c|c|}
\hline & $\begin{array}{l}\text { Early } \\
\text { Cohort: } \\
<12 \\
\text { months }\end{array}$ & $\begin{array}{c}\text { Mid } \\
\text { Cohort: } \\
12-60 \\
\text { months }\end{array}$ & $\begin{array}{c}\text { Late } \\
\text { Cohort: } \\
>60 \\
\text { months }\end{array}$ \\
\hline Age & 52.0 & 48.6 & 49.8 \\
\hline$\%$ Male & 64.5 & 59.8 & 59.9 \\
\hline$\%$ asthmatic & 20.1 & 28.8 & 36.6 \\
\hline$\%$ smokers & 22.6 & 22.4 & 17.9 \\
\hline$\%$ patient reported allergies & 23.1 & 33.1 & 39.2 \\
\hline$\%$ aspirin sensitivity & 0.6 & 1.6 & 1.4 \\
\hline$\%$ CRSwNP & 67.1 & 60.9 & 68.4 \\
\hline \multicolumn{4}{|l|}{ Polyp grade (\%) } \\
\hline 1 & 20.9 & 28.4 & 25.3 \\
\hline 2 & 51.3 & 38.9 & 44.3 \\
\hline 3 & 27.8 & 32.7 & 30.4 \\
\hline Mean Lund-Mackay Score & 9.6 & 10.2 & 11.1 \\
\hline Mean SNOT-22 & 35.8 & 39.7 & 40.8 \\
\hline
\end{tabular}

All patient groups demonstrated improvement in SNOT-22 scores at all time points post-operatively. The average SNOT-22 by time point and cohort and the percentage changes in SNOT-22 are shown in Table 3.

Preoperatively, the average SNOT-22 scores ranged from 35.3 (all patients) or 33.7 (patients with asthma excluded) in the Early group to 40.8 (all patients) or 40.6 (patients with asthma excluded) in the Late group. Post-operatively, there was a significant decrease in average SNOT-22 scores across all patient groups (paired T test, $\mathrm{p}<0.001$ for each group compared to baseline at each time point). Mean SNOT-22 scores were lowest at all time points in the Early cohort versus the Mid and Late cohorts (Table 3). To determine whether changes were simply a reflection of preoperative status, absolute and percentage change from baseline were also calculated. The range of absolute score changes from baseline across all three cohorts was surprisingly narrow, from an average 18.6 points in the Early group to 17.3 in the Late group, with no significant difference between groups. This range remained narrow at 12 months post-operatively but broadened at 60 months, with the Late cohort showing signs of increasing SNOT-22 scores (mean absolute score change 16.8 for Early Cohort and 11.7 for Late Cohort). Percentage changes from baseline were greater for the Early cohort than the Late cohort at all time points. This reached statistical significance $(p<0.005)$ 
Table 3. Absolute values for SNOT-22 at each time point, actual change in SNOT-22 score from baseline, and percentage change from baseline, by duration of symptoms. Repeated analysis after excluding asthmatic patients shown in italics. One way analysis of variance used to test for difference between groups for total value and absolute change in score. (Change score calculated only for patients with paired data, and therefore differs slightly from simple difference in means at each time point. Percentage change calculated for each individual - mean percentage change is then reported, not the percentage change of the difference in means).

\begin{tabular}{|c|c|c|c|c|c|c|c|c|c|c|c|}
\hline $\begin{array}{c}\text { Duration of symptoms before } \\
\text { surgery }\end{array}$ & $\mathbf{n}$ & $\begin{array}{c}\text { Mean } \\
\text { Pre-op } \\
\text { SNOT- } \\
22 \\
\text { (SE) }\end{array}$ & $\begin{array}{c}\text { Mean } 3 \\
\text { month } \\
\text { SNOT- } \\
22 \\
\text { (SE) }\end{array}$ & $\begin{array}{l}\text { Mean } \\
12 \\
\text { month } \\
\text { SNOT- } \\
22 \\
\text { (SE) }\end{array}$ & $\begin{array}{c}\text { Mean } \\
60 \\
\text { month } \\
\text { SNOT- } \\
22 \\
(\text { SE) }\end{array}$ & $\begin{array}{c}\text { Mean } \\
\text { Change } \\
\text { in } \\
\text { SNOT- } \\
22 \text { at } 3 \\
\text { months } \\
\text { (SE) }\end{array}$ & $\begin{array}{l}\text { Change } \\
\text { in } \\
\text { SNOT- } \\
22 \text { at } 12 \\
\text { months }\end{array}$ & $\begin{array}{l}\text { Change } \\
\text { in } \\
\text { SNOT- } \\
22 \text { at } 60 \\
\text { months }\end{array}$ & $\begin{array}{c}\% \\
\text { Change } \\
\text { in } \\
\text { SNOT- } \\
22 \text { at } 3 \\
\text { months } \\
\text { (SE) }\end{array}$ & $\begin{array}{c}\% \\
\text { Change } \\
\text { in } \\
\text { SNOT- } \\
22 \text { at } 12 \\
\text { months }\end{array}$ & $\begin{array}{c}\% \\
\text { Change } \\
\text { in } \\
\text { SNOT- } \\
22 \text { at } 60 \\
\text { months }\end{array}$ \\
\hline$<12$ months & $\begin{array}{l}172 \\
139\end{array}$ & $\begin{array}{l}35.3 \\
(0.7)\end{array}$ & $\begin{array}{l}18.0 \\
(1.6)\end{array}$ & $\begin{array}{l}19.5 \\
(1.8)\end{array}$ & $\begin{array}{l}19.7 \\
(2.2)\end{array}$ & $\begin{array}{l}18.6 \\
(1.8)\end{array}$ & $\begin{array}{l}17.2 \\
(1.8)\end{array}$ & $\begin{array}{l}17.3 \\
(2.1)\end{array}$ & $\begin{array}{l}43.5 \\
(5.7)\end{array}$ & $\begin{array}{l}43.3 \\
(6.0)\end{array}$ & $\begin{array}{l}46.1 \\
(5.9)\end{array}$ \\
\hline & & 33.7 & 18.2 & 19.9 & 17.3 & 17.2 & 15.7 & 18.2 & & & \\
\hline $12-60$ months & $\begin{array}{l}750 \\
567\end{array}$ & $\begin{array}{l}39.7 \\
(0.8) \\
38.7\end{array}$ & $\begin{array}{l}22.9 \\
(0.8) \\
\\
21.7\end{array}$ & $\begin{array}{l}24.9 \\
(0.9)\end{array}$ & $\begin{array}{l}25.0 \\
(1.0)\end{array}$ & $\begin{array}{l}17.3 \\
(0.8)\end{array}$ & $\begin{array}{l}15.2 \\
(0.9) \\
\\
15.7\end{array}$ & $\begin{array}{l}13.6 \\
(1.1) \\
13.9\end{array}$ & $\begin{array}{l}39.4 \\
(2.2)\end{array}$ & $\begin{array}{l}34.1 \\
(2.4)\end{array}$ & $\begin{array}{l}28.2 \\
(3.3)\end{array}$ \\
\hline$>60$ months & $\begin{array}{l}571 \\
406\end{array}$ & $\begin{array}{r}40.8 \\
(0.5) \\
\\
40.6\end{array}$ & $\begin{array}{l}24.4 \\
(0.9) \\
\\
24.8\end{array}$ & $\begin{array}{l}26.3 \\
(1.0)\end{array}$ & $\begin{array}{l}28.2 \\
(1.2)\end{array}$ & $\begin{array}{l}17.3 \\
(0.9)\end{array}$ & $\begin{array}{l}14.5 \\
(0.9)\end{array}$ & $\begin{array}{l}11.7 \\
(1.3) \\
\\
11.9\end{array}$ & $\begin{array}{l}37.0 \\
(3.1)\end{array}$ & $\begin{array}{l}30.8 \\
(4.3)\end{array}$ & $\begin{array}{l}16.6 \\
(5.6)\end{array}$ \\
\hline One-way ANOVA & & $\begin{array}{c}F=5.2 \\
p<0.006\end{array}$ & $\begin{array}{c}F=5.9 \\
p<0.003\end{array}$ & $\begin{array}{c}F=5.1 \\
p<0.006\end{array}$ & $\begin{array}{c}F=6.0 \\
p<0.002\end{array}$ & $\begin{array}{l}F=0.27 \\
p=0.64\end{array}$ & $\begin{array}{l}F=0.90 \\
p=0.29\end{array}$ & $\begin{array}{l}F=2.37 \\
p=0.49\end{array}$ & $\begin{array}{l}F=0.66 \\
p=0.5\end{array}$ & $\begin{array}{l}F=1.42 \\
p=0.24\end{array}$ & $\begin{array}{l}F=5.18 \\
p<0.005\end{array}$ \\
\hline
\end{tabular}

at 60 months (Early $46.1 \%$, Mid $28.2 \%$, Late $16.6 \%$ ). While improvements were maintained from 12 to 60 months in the Early cohort, a progressive deterioration of symptom scores findings are shown visually in Figure 1, which demonstrates increasing divergence as the follow-up period after surgery increases.

\section{Percentage change in SNOT-22 according to sympton duration prior} to first surgery

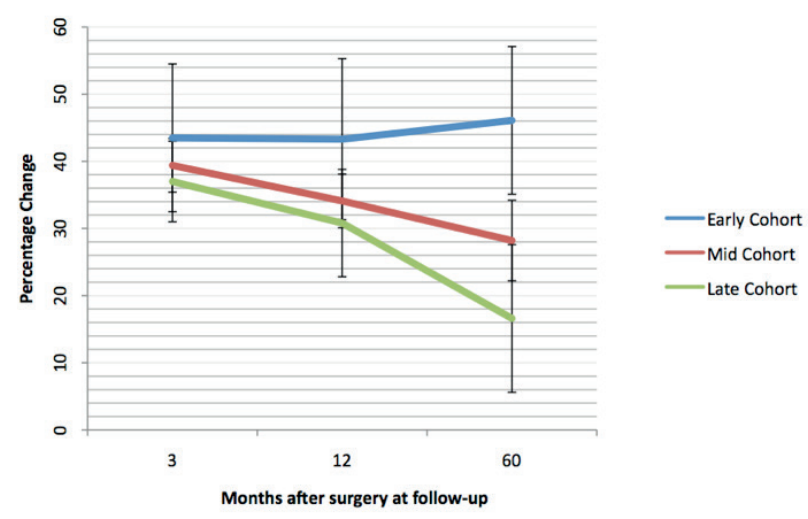

Figure 1. Percentage change in SNOT-22 for each cohort at 3,12 and 60 months (95\% confidence intervals shown).
Repeating the analyses above but excluding asthmatic patients demonstrated the same pattern of results, suggesting that the higher rates of asthmatic patients in the Late cohort did not confound the results (Table 3, results shown in italics). Further exclusion of patients with allergies did not result in a change in the pattern of results. At 60 months there was a 59.0\% improvement in SNOT-22 score from baseline in the early group, 35.6\% in the Mid Cohort, and $31.6 \%$ in the late cohort.

The percentage of patients achieving at least an 8.9-point difference in SNOT-22 score from preoperative to post-operative time points (the MCID) is shown in Table 4. At 3 months postoperatively, $75.0 \%, 74.5 \%$ and $75.4 \%$ of patients in the Early, Mid and Late cohorts reached the MCID, respectively $(p=0.971)$. At 12 months, however, $78.0 \%$ of the Early cohort maintained a MCID in pre-post SNOT-22 scores versus $70.8 \%$ and $70.5 \%$ in the Mid and Late cohorts respectively, and by 60 months, $71.5 \%$ of the Early cohort versus $57.3 \%$ of the Mid and $53.0 \%$ of the Late cohort reached the MCID - this difference was significant $(p=$ 0.028).

Multivariate regression confirmed that duration of symptoms to surgery remained an important predictor of post-operative outcomes when other demographic factors (pre-operative 
Table 4. Percentage of patients achieving MCID by symptom duration.

\begin{tabular}{|c|c|c|c|c|}
\hline Cohort & $\begin{array}{l}\text { Chi test } \\
\text { for } \\
\text { difference } \\
\text { between } \\
\text { groups }\end{array}$ & $\begin{array}{c}\text { Early } \\
\text { Cohort: } \\
<12 \\
\text { months }\end{array}$ & $\begin{array}{l}\text { Mid } \\
\text { Cohort: } \\
12-60 \\
\text { months }\end{array}$ & $\begin{array}{l}\text { Late } \\
\text { Cohort: } \\
>60 \\
\text { months }\end{array}$ \\
\hline Follow-up & & \multicolumn{3}{|c|}{$\%$ patients achieving MCID of 8.9} \\
\hline 3 months & $p=0.971$ & 75.0 & 74.5 & 75.4 \\
\hline 12 months & $p=0.05$ & 78.0 & 70.8 & 70.5 \\
\hline 60 months & $p=0.028$ & 71.2 & 57.3 & 53.0 \\
\hline
\end{tabular}

SNOT-22, LM score, age, gender, asthma and allergy) and extent of surgery were controlled for (at 12 months post-operatively $\beta=2.67, p=0.03$; at 60 months post-operatively $\beta=3.59, p=$ 0.05).

\section{Discussion}

With austerity measures becoming widespread, there is a drive to manage referral pathways, with the potential to restrict access to secondary care and surgical management. This is likely to both reduce and delay referrals, with many commissioning guidelines insisting on prolonged trials of medical therapy in primary care. While these measures may have an immediate budgetary impact, it is important to carefully consider the consequences of such decisions on patients' quality of life and future treatment success, as long-term treatment costs and lost productivity may outweigh short-term gains.

In this study, we evaluated whether the clinical benefits of sinus surgery varied according to the duration of CRS symptoms prior to surgery in patients treated either within 12 months of symptom onset (Early cohort), between 12-60 months of symptoms (Mid cohort) or more than 60 months from first symptoms (Late cohort). Clinical benefits were calculated based on post-operative quality of life outcome scores, using a validated instrument for sinusitis (SNOT-22 scores). Our results indicate that absolute SNOT-22 scores were significantly lower in the Early cohort at all pre- and post-operative time points versus those of the Mid and Late cohorts. Percentage changes in scores between groups from preoperative to post-operative time points demonstrated a significant trend of greater change for patients in the Early cohort as duration of follow-up increased. In addition, when analysing the percentage of patients reaching the MCID for SNOT-22, there was a significantly greater proportion of patients in the Early and Mid cohorts reaching the MCID compared with the Late cohort at 12 and 60 months post-operatively. Our results therefore suggest that intervention within 12 months of the onset of symptoms may yield better clinical outcomes in terms of patient-reported quality of life, at least as far as 5 years post-surgery.

Recent guidelines recommend that surgery should be considered if a 3 month trial of medical treatment fails to bring about adequate improvement in symptom levels ${ }^{(4)}$. However, based on the National Comparative Audit data analysed herein, in the UK $88.2 \%$ of patients have symptoms for one year or more prior to first-time surgery, and $38.2 \%$ of the cohort are symptomatic for more than 5 years. We do not know for how long these patients have been receiving medical treatment for their sinusitis, but it is likely that the 3-month period recommended by the EPOS 2012 Guidelines ${ }^{(4)}$ for medical management is far exceeded in the vast majority of cases.

Preoperatively, patients in the Early cohort had statistically, but not clinically, lower average SNOT-22 scores compared to the Mid and Late cohorts. The average difference in SNOT-22 scores between the Early and Late cohort was 5.0 points and as described previously, the MCID for SNOT-22 is 8.9 points. Therefore, while the Early cohort may have scored lower on the SNOT-22, their perception of symptoms was not clinically different from that of patients in the Mid and Late cohorts. Patients in the Early cohort also had less severe radiological disease, as shown by the LM score. While the LM score does not necessarily correlate with subjective, patient-reported symptoms, it is a meaningful indicator of disease severity and has been shown to be associated with post-operative outcome ${ }^{(10)}$. There is little published regarding the natural history of CRS, but our results, demonstrating increasing preoperative SNOT-22 and LM scores from the Early to Mid and Late cohorts, suggest that both radiological and symptomatic disease severity increases with prolonged duration of symptoms.

Following surgery, all patients experienced significant symptomatic improvement as shown by the SNOT-22. This finding is consistent with other recent studies demonstrating the effectiveness of sinus surgery ${ }^{(6)}$. Patients treated within 12 months of symptom onset had, on average, statistically lower post-operative SNOT-22 scores versus the other cohorts, at all post-operative time points. In addition, the procedure was found to have a durable effect, especially in the Early cohort where more than $70 \%$ of patients maintained a clinically significant improvement from baseline as far as 60 months post-operatively. In the Late cohort, although $75 \%$ of patients obtained a clinically significant improvement from surgery as determined 3 months post-operatively, this number gradually decreased to $53 \%$ at 60 months post-operatively. These findings suggest that early intervention may increase durability of the treatment.

Limitations of this study include general methodology limitati- 
ons as expected with any audit or registry, i.e. no randomisation was done to ensure that probability of prognosis was equal across all three groups. Therefore, and as discussed above, differences in comorbidity rates across groups need to be considered. We are reliant upon patient reported duration of symptoms, and it is possible that some patients were unable to differentiate symptoms of co-existing allergic rhinitis from those of CRS, both before and after surgery. We have attempted to control for this by repeating our analysis having excluded thise with asthma and allergies, and by performing a multivariate analysis, but some bias may persist. An additional limitation may be related to the outcomes tool; patient-reported outcomes, even validated ones, may have some intrinsic variability and, while still one of the best predictors of patient well being, may differ from clinical or radiographic outcomes. Finally, and as with most observational cohort studies, there was a progressive loss of respondents with time. This is due in part to general loss to follow-up despite 2 attempts with postal questionnaires as well as, in a small number of cases, withdrawal of consent to further contact. However, a nearly $80 \%$ response rate was achieved at 12 months, with no difference in drop-out rates between the groups of interest. The greatest differences between groups were found at 60 months, when response rates were at their lowest. There is therefore a risk of bias due to loss to follow-up. Accepting the limitations of this current study, we therefore plan to test the same hypothesis using a second independent patient cohort, the Clinical Practice Research Datalink (CPRD) database.

It is interesting that there were much higher rates of asthma and allergy in the Late cohort. While endoscopic sinus surgery is aimed at relieving sinonasal symptoms, it has also been shown to improve bronchial symptoms and reduce medication use for asthma ${ }^{(11-13)}$, and therefore this group may potentially benefit even more from surgical intervention if medical treatment has failed, when compared with non-asthmatic patients. It is unclear why surgery was delayed in a large proportion of these patients: all patients were considered to be at an American Society of Anaesthesiology (ASA) Physical Status Score grade 2 or less at the time of the surgery so these patients did not have an increased surgical risk. We repeated all analyses without the asthmatic patients, to identify any confounding effects. Not surprisingly, analyses showed similar trends irrespective of whether asthmatic patients were included or excluded. This may be due to the fact that, while asthmatic patients may experience greater healthcare needs and general morbidity, their self-reported perceptions of disease symptoms and benefits from surgery was shown in prior research to be similar to that of non-asthmatic patients ${ }^{(14)}$.

In the consolidated 3,128-patient audit results, pre-operative
SNOT-22 scores were found to be the greatest predictor of postoperative outcomes, as patients with higher scores achieved greater absolute reductions in SNOT-22 scores - on average, a halving of their pre-operative score. In our study, however, when patients were subdivided into 3 cohorts based on the preoperative duration of symptoms, the opposite was observed: the Early cohort achieved greater absolute and relative reductions in symptom scores than both the Mid and Late cohorts, despite starting with lower scores. Moreover, the Early cohort's postoperative symptom scores remained low and constant over the entire 5-year post-operative period, whereas progressive decline in improvements was noticed in the other groups, particularly the Late cohort. These results suggest that the maximum and most persistent benefit from endoscopic sinus surgery occurs in patients undergoing surgery at an early stage in their history of CRS disease, in keeping with current guidelines. The multivariable regression analysis further confirmed that preoperative duration of symptoms was an important predictor of surgical outcome. Delays in surgical intervention, where it is indicated, may therefore adversely affect outcome.

There are many possible reasons why earlier surgical intervention improves outcome. Surgery leads to improved ventilation of the sinuses and allows better irrigation and instillation of topical steroids.; it may therefore be that earlier surgery simply allows medical therapy to be more effective. However, surgery may help by removing factors that adversely affect outcome. Bacterial biofilms are known to be associated with CRS, and are thought to contribute to the persistent inflammatory state ${ }^{(15)}$. Endoscopic sinus surgery has been shown to significantly reduce biofilm density, with associated improvements in QOL and objective outcome measures ${ }^{(16)}$. Osteitis is associated with more severe inflammation and worse disease severity scores. The natural history of osteitis in CRS in not known, but its presence is associated with an increase in the number of surgical procedures undertaken; further studies are needed to identify whether earlier surgical intervention and removal of diseased bone may prevent disease progression ${ }^{(17)}$. There is also increasing evidence that irreversible mucosal changes may occur in CRS, in direct correlation to the duration of the disease ${ }^{(18)}$. Whilst steroids, due to their anti-inflammatory properties, have some effect on this remodeling process, it has been proposed that early surgical intervention to reduce the inflammatory load may be beneficial in preventing disease progression ${ }^{(7)}$.

Whilst we suggest that ongoing untreated sinusitis leads to disease progression with mucosal remodelling and accumulation of adverse features such as biofilms and osteitis, it is possible that prolonged use of topical or systemic medications may also be detrimental to long term outcomes. It is beyond the scope of 
the current study to identify the mechanisms behind the differences in outcome; future studies will be needed if our findings are replicated in independent cohorts.

Attempts to reduce healthcare expenditure by restricting access to secondary care should therefore be carefully considered, as such measures may have a negative and lasting impact on patients' ability to experience meaningful improvements from CRS symptoms. While there is a clear ethical consideration in denying relief to these patients, the societal impact of CRS should also be considered against any potential short-term cost-saving measure. Recurrent disease incurs direct costs from ongoing health care utilisation. Indirect costs are likely to be far greater; productivity analyses of patients suffering from CRS have recently been evaluated and shown to be more than $30 \%$ lower than that of patients without CRS ${ }^{(19)}$. In patients with CRS, productivity at work improved by approximately $76 \%$ after surgery. Prompt referral allowing correct diagnosis to be reached and a subsequent trial of maximum medical therapy will allow surgical candidates to be identified at an earlier stage than we currently achieve. Improving outcomes from surgery will reduce both direct and indirect long term costs of CRS.

\section{Conclusion}

Maximum medical therapy should form the first-line of care for patients with CRS, but both our results and those of Smith et al. ${ }^{(5,6)}$ suggest that when this approach has failed, surgery is best considered without significant further delay. In addition, our study indicates that delaying surgical intervention may reduce both the extent of symptomatic benefit from surgery, and significantly reduce the percentage of CRS patients who experience sustained clinical improvements. Clinical improvement as defined by SNOT-22 was stable in patients treated early on, for at least the 60 months post-operative period reported herein. This is the first published evidence suggesting that delaying endoscopic sinus surgery in CRS patients refractory to medical management may lead to worse clinical outcomes than when surgery is offered at an earlier stage in the history of the disease.

Timely assessment, an appropriate trial of medical therapy and evaluation of the response to treatment will allow us to treat our patients in the time frame recommended by current guidelines ${ }^{(20)}$, while delays in this pathway may be detrimental to long term outcomes.

\section{Acknowlegdement}

The authors acknowledge Chantal Holy, PhD, for editorial support.

\section{Authorship contribution}

$\mathrm{CH}$ : study design, data analysis, preparation of manuscript $J R$ : Preparation of manuscript

VJL: study design, editorial input

\section{Conflicts of Interest}

None reported

\section{References}

1. Hastan D, Fokkens WJ, Bachert C, Newson RB, Bislimovska J, Bockelbrink A, et al. Chronic rhinosinusitis in Europe--an underestimated disease. A GA(2)LEN study. Allergy. 2011; 66: 1216-1223.

2. Gliklich RE, Metson R. The health impact of chronic sinusitis in patients seeking otolaryngologic care. Otolaryngol Head Neck Surg. 1995; 113: 104-109.

3. Pleis JR, Lethbridge-Cejku M. Summary health statistics for U.S. adults: National Health Interview Survey, 2005. Vital Health Stat 10. 2006; 232: 1-153.

4. Fokkens WJ, Lund VJ, Mullol J, Bachert C Alobid I, Baroody F, et al. European Position Paper on Rhinosinusitis and Nasal Polyps 2012. Rhinol Suppl. 2012 (23): 1-298

5. Friedman M, Hamilton C, Samuelson CG Maley A, Wilson MN, Venkatesan TK, et al. Dead Sea salt irrigations vs saline irrigations with nasal steroids for symptomatic treatment of chronic rhinosinusitis: a randomized, prospective double-blind study. Int Forum Allergy Rhinol. 2012; 2: 252-257.

6. Jervis-Bardy J, Wormald PJ. Microbiological outcomes following mupirocin nasal washes for symptomatic, Staphylococcus aureuspositive chronic rhinosinusitis following endoscopic sinus surgery. Int Forum Allergy Rhinol. 2012: 2: 111-115.

7. Bassiouni A, Chen PG, Wormald PJ. Mucosal remodeling and reversibility in chronic rhinosinusitis. Curr Op Allergy Clin Immunol. 2013; 13: 4-12.

8. Hopkins $C$, Browne JP, Slack R, Lund V, Topham J, Reeves B, et al. The national comparative audit of surgery for nasal polyposis and chronic rhinosinusitis. Clin Otolaryngol. 2006; 31: 390-398

9. Hopkins C, Gillett S, Slack R, Lund VJ, Browne JP. Psychometric validity of the 22-item Sinonasal Outcome Test. Clin Otolaryngol. 2009; 34: 447-454.

10. Hopkins C, Browne JP, Slack R, Lund V, Brown P. The Lund-Mackay staging system for chronic rhinosinusitis: how is it used and what does it predict? Otolaryngol Head Neck Surg. 2007; 137: 555-561.

11. Dhong HJ, Jung YS, Chung SK, Choi DC Effect of endoscopic sinus surgery on asthmatic patients with chronic rhinosinusitis.
Otolaryngol Head Neck Surg. 2001; 124: 99-104.

12. Proimos $\mathrm{E}$, Papadakis CE, Chimona TS, Kiagiadaki D, Ferekidis E, Yiotakis J. The effect of functional endoscopic sinus surgery on patients with asthma and CRS with nasal polyps. Rhinology. 2010; 48: 331-338.

13. Ragab S, Scadding GK, Lund VJ, Saleh H. Treatment of chronic rhinosinusitis and its effects on asthma. Eur Respirat J. 2006; 28: 68-74.

14. Smith TL, Mendolia-Loffredo S, Loehrl TA, Sparapani R, Laud PW, Nattinger AB. Predictive factors and outcomes in endoscopic sinus surgery for chronic rhinosinusitis. Laryngoscope. 2005; 115: 2199-2205.

15. Harvey RJ, Lund VJ. Biofilms and chronic rhinosinusitis: systematic review of evidence, current concepts and directions for research. Rhinology. 2007; 45: 3-13

16. Hai PV, Lidstone C, Wallwork B. The effect of endoscopic sinus surgery on bacterial biofilms in chronic rhinosinusitis. Otolaryngol Head Neck Surg. 2010; 142 (3 Suppl 1): S2732.

17. Georgalas C. Osteitis and paranasal sinus 
inflammation: what we know and what we do not. Curr opin Otolaryngol Head Neck Surg. 2013; 21: 45-49.

18. Rehl RM, Balla AA, Cabay RJ, Hearp ML, Pytynia KB, Joe SA. Mucosal remodeling in chronic rhinosinusitis. Am J Rhinol. 2007; 21:651-657.

19. Stankiewicz J, Tami T, Truitt T, Atkins J, Winegar B, Cink P, et al. Impact of chronic rhinosinusitis on work productivity through one-year follow-up after balloon dilation of the ethmoid infundibulum. Int Forum Allergy Rhinol. 2011; 1: 38-45.

20. Fokkens WJ, Lund VJ, Mullol J, Bachert C Alobid I, Baroody F, et al. EPOS 2012 European position paper on rhinosinusitis and nasal polyps 2012. A summary for otorhinolaryngologists. Rhinology. 2012; 50: 1-12.
Claire Hopkins, BM BCh MA (Oxon)

DM FRCS (ORL-HNS)

ENT Dept, Guy's Hospital

London SE1 9RT

United Kingdom

Tel: +44 -20-7188 2215

Fax: +44-20-7188 2206

E-mail address:

clairehopkins@yahoo.com 